\title{
Las estructuras científico-burocráticas y los "Ismos" de la investigación en las ciencias sociales
}

\section{Brigitte Bernard*}

\section{Resumen}

En este trabajo, proponemos una reflexión sobre el papel conservador y desmovilizador de las estructuras científico-burocráticas, y de sus parámetros de evaluación, entre los que juegan un papel destacado, las revistas arbitra das, de nivel "altamente científico". El modelo positivista de ciencia que prevalece en el ámbito universitario, ha generado una concepción fisicista que oculta las relaciones de poder, y promueve la estabilización del sistema político-ideológico y la alienación de la comunidad científica. Además, el cientificismo como parte de la cultura cientifica imperante, la cual al explicar el subdesarrollo por el atraso científico-tecnológico, justifica la priorización de la investigación en las áreas naturales y tecnológicas a la vez que desactiva la capa cidad crítica y esclarecedora de las ciencias sociales.

Palabras clave: Ciencias sociales, investigación, cientificismo, burocracia.

- Investigadora del Instituto de Filosofia del Derecho "Dr. J.M. Delgado O." La Universidad del Zulia - Maracaibo - Venezueia. 


\title{
The Scientific Bureaucratic Structures and the "Isms" of Research in the Social Sciences
}

\begin{abstract}
This paper proposes some thoughts on the conservative and demobilizing role of scientific bureaucratic structures, and their parameters for evaluation, among others, those which play a fundamental role, the "highly scientific" arbitrated journals. The positivist model of science which prevails in the university environment has generated a physicistic concept which covers up power relations, and promotes the stabilization of the ideological-political system, and the alienation of the scientific community. Furthermore, scientificism", as part of the dominant scientific culture, which explains under-development as scientific and technological backwardness, justifies giving priority to research in the areas of natural sciences and technology and deactivates the critical and aclaratory capacity of the social sciences.
\end{abstract}

Key words: Social science, Research, Scientificism, Bureaucratic.

\section{Introducción}

En las últimas décadas, la investigación en las ciencias sociales en América Latina, se ha caracterizado por un retorno al positivismo y al empirismo inductivo como concepción dominante en los trabajos publicados en las revistas especializadas. Se ha abandonado la posición militante y comprometida de los años 60 y 70 en aras de un regreso a la emulación de las ciencias de la naturaleza, en una renovación de una fe un tanto anacrónica en la ciencia como paradigma de la modernidad (Popper, 1994:25-27; de Sousa Santos, 1996:202), se asiste a la promoción de una supuesta cultura científica que no es mas que la reedición de la concepción positivista decimonónica.

En el trabajo que presentamos a continuación, proponemos una reflexión sobre el papel conservador y desmovilizador de las estructuras científico-burocráticas y de sus parámetros de evaluación, entre los que juega un papel destacado, la publicación en revistas arbitradas, de nivel "altamente científico", de los resultados de la investigación. En este sentido, consideramos que constituiría una materia primordial de esa tarea de investigación, el definir hasta qué punto los mencionados parámetros contribuyen a distorsionar la actividad cientifica en el área de las ciencias sociales, al adoptar en nombre de la rigurosidad y de la objetividad, un enfoque aséptico, positivo, factual y funcional de la realidad social (Goldmann, 1977:21).

A los efectos indicados, nos referiremos en primer lugar, al modelo positivista de ciencia que prevalece en el ámbito universitario, el cual al término de una 
lucha secular en favor de la especificidad de las ciencias humanas, logró imponer una concepción fisicista en la que permanecen ocultos los resortes que determinan las relaciones de poder (Rojas Soriano, 1983:57). En segundo lugar, mostraremos como dicho concepto de ciencia está estrechamente vinculado a la ideología dominante y que sus implicaciones son estabilizadoras del sistema políticoideológico y alienantes respecto de la comunidad científica inmersa en los procesos de conocimiento que se generan a su amparo (Bernard, 1978:33; 1989:8-21).

Finalmente, en una tercera parte de este trabajo, nos referiremos al cientificismo como concepción subyacente a la cultura científica imperante, la cual al explicar el subdesarrollo por el atraso científico-tecnológico, exime al sistema nacional e internacional de relaciones de poder, de toda responsabilidad respecto del mismo, en tanto justifica la priorización de la investigación en las áreas naturales y tecnológicas a la vez que desactiva la capacidad crítica y esclarecedora de las ciencias sociales (Delgado,1987:112; 1989: 33; Bernard, 1995a).

En conclusión, es necesario analizar el papel de la organización científicoburocrática del establecimiento económico y político, la cual a través de la revista arbitrada, asi como de otros instrumentos de control ideológico como el de la carrera del investigador, el PPI, el financiamiento de proyectos para el sistema productivo, la promoción de la participación en congresos científicos, fomenta la adhesión de la comunidad científica del Tercer Mundo, al concepto de economia mundial globalizada como estrategia de oculta miento de la dependencia (Terrades, 1977; Delgado, 1990).

\section{Clencia, positivismo y objetividad}

El concepto de ciencia entendido entre otras tantas conceptualizaciones, como "un conjunto de conocimientos con base lógica, método propio y objeto determinado que permite la previsión" (Nuñez Tenorio,1972), surge con el auge en los siglos XVI y XVII, de las ciencias naturales.

La política y el Derecho que desde antiguo, habian alcanzado niveles cimeros de desarrollo, nunca hasta entonces se habían planteado una reflexión epistemológica en torno a su quehacer. Las ciencias de la naturaleza se les adelantaron gracias a su desarrollo espectacular en los campos de la astronomía y de la fisica, con Galileo y Newton (Freund, 1975). Es cuando surge el llamado método "científico", o método empirico-inductivo que permite la formulación de leyes generales a partir del examen protocolar de casos o experimentos concretos estadísticamente significativos (Sanchez) Guarisma, s/f:19).

El científico de la naturaleza, el físi$\mathrm{co}$, el químico, el biólogo, el zoólogo, el botánico, etc, descubrieron que al establecer en la experiencia, relaciones constantes de causalidad o al desagregar un todo en sus partes y vice-versa, al recomponer esa totalidad a partir de sus elementos, estaban en condición de explicar los fenómenos de la naturaleza por medio de afirmaciones asertóricas basadas en verdades de hecho comprobadas experimentalmente (Cossio, 1959:35-37). 
Otro tanto hicieron los matemáticos y los lógicos, al descubrir que en su respectiva área, no requerían siquiera acudir al método experimental, sino que por via racional-deductiva, podian a partir de la formulación de principios generales $y$ axiomas, derivar juicios apodícticos fundados en verdades de razón. El que $2+2$ $=4$ en todos los casos, responde a una verdad basada en el principio de no-contradicción, de la misma manera que la imposibilidad de vivir quinientos años para un ser humano constituye una verdad de hecho irrefutable; pero "las verdades de razón' son juicios apodícticos, es decir que son asi y no pueden dejar de ser así, porque no podrían ser de otra manera... por eso tienen una fuerza interna que nos obliga, queramos o no, a aceptalas" (Cossio, 1995, 31).

Con la consagración de dichas ciencias, había nacido el "positivismo científico" que Auguste Comte plantearía como modelo a todos los ámbitos científicos, en la convicción de que no obstante la particularidad de las ciencias de la cultura, dicha corriente epistemológica tenía mucho que aportar a las mismas en los casos en los que ello resultara pertinente En esta forma, Comte proponía eliminar del conocimiento científico de lo social, el influjo de la metafísica y de la religión, adoptando ante la realidad, lo que habia denominado "positivismo sociológico", es decir la actitud consistente en la búsqueda de la verdad acerca de los fenómenos sociales, a partir del uso de la metodología de las ciencias naturales amen de otras propias al ámbito de lo humano (Freund. 1975: 67; Bernard, 1995:128).
Hoy sabemos que la diferencia entre las condiciones de trabajo de los 'físicos, químicos y fisiólogos' y las de los sociólogos o los historiadores, no es de grado sino de naturaleza; en el punto de partida de la investigación física o química, hay un acuerdo real e implícito entre todas las clases que constituyen la sociedad actual, acerca del valor, la naturaleza y el fin de la investigación... En cambio, en las ciencias humanas hallamos diferencias radicales de actitud, anteriores al comienzo del trabajo de investigación, y que con frecuencia, quedan implicitas e inconscientes" (Goldmann, 1977, 25-26).

Ahora bien, no obstante los ingentes esfuerzos realizados en forma sistemática desde el siglo pasado, por las ciencias que Rickert denominaba "culturales", a partir de la afirmación de Dilthey para quien "explicamos a la naturaleza y comprendemos a la sociedad", o la de Windelband para quien no debia confundirse realidad y naturaleza, se han realizado esfuerzos dirigidos a mostrar la diferencia esencial entre los enfoques nomotéticos de las ciencias naturales e idiográficos de las ciencias humanas cuyo objeto se caracteriza por su sentido valorativo y su esencial historicidad (Freund, 1975).

Decíamos al inicio de nuestra Introducción, que no obstante dichos esfuerzos, desde hace ya varias décadas, asistimos a una renovación de la perspectiva positivista empirista en las ciencias sociales. En efecto, bástanos leer la bibliografía en el campo de la Metodología en las ciencias sociales, para observar que el método científico originario de las ciencias naturales, ha sido objeto de una ex- 
trapolación masiva a las ciencias sociales, hasta el punto de afirmarse que "el concepto de contrastabilidad (testability)... es el núcleo de la cientificidad..., ya que una idea puede considerarse científica solo si es objetivamente contrastable con datos empíricos" (Bunge, 1980:31).

El Empirismo que en las ciencias sociales, cobra auge en los Estados Unidos a partir de 1930, destaca entre sus principales exponentes, a Paul Lasarsfeld, comparte con el Positivismo y el método inductivo, una perspectiva fáctica de la realidad, (sea natural o social), la cual "conciben en términos de fenómenos", externos al investigador quien, para ser objetivo, "no debe incluir sus nociones, ideas o valores, (sino) limitarse a captar el objeto de estudio, tal y como se presenta en la realidad, a través de los pasos, etapas proce dimientos y técnicas que proporciona el método científico" (Méndez, 1984:180-181).

Debido a que los fenómenos tanto sociales como naturales presentan propiedades que se relacionan entre sí, se conciben dichas relaciones como ausales, enmarcadas en el "principio de la legalidad de la naturaleza y de la sociedad. Todo está sometido a un orden establecido previamente, es decir, a unas leyes generales que el investigador debe descubrir" (Méndez, 1984: 182).

\section{Clencias sociales, fisicismo e ideologia}

Como consecuencia de la supuesta constatación de una legalidad social fáctica, el Positivismo empirista e inductivo propugna una concepción estática y fragmentaria de la realidad, la cual produ- ce la ilusión de un mundo quieto e inmune a los cambios históricos, en tanto su conceptualización se limita a una formulación general de lo observable, de la que está ausente cualquier intento de interpretación teórica que no sea la transformación en principio de explicación, de las constantes observadas a nivel empírico, $u$ en otros términos, la consagración de los fenómenos descritos como inmutables (Bernard, 1989:20-21).

En razón de ello, "la filosofía positiva se erigió, en su momento histórico, en defensora del ca- pitalismo por considerarlo una situación eterna, única, con lo cual trataba de evitar el surgimiento de doctrinas y prácticas socio-políticas que señalara 10 relativo de su postura asi como la concepción ideológica subyacente en el estudio de la realidad social" (Rojas Soriano, 1983:57).

Las implicaciones ideológicas del Positivismo están particularmente marcadas en una de las corrientes metodológicas derivadas del mismo, objeto de especial predilección en las ciencias sociales, como lo es el funcionalismo. En efecto, según Lowy, "todavia hoy, corrientes manifiestamente neopositivistas ejercen una influencia decisiva, si no hegemóni$\mathrm{ca}$, en las ciencias sociales universitarias, académicas.., particularmente en los Estados Unidos. Evidente mente, sus formas han cambiado : conductismo y funcionalismo" (Rojas Soriano, 1983:58).

En este sentido, recordemos que el funcionalismo concibe a la sociedad como un todo orgánico cuyas estructuras y relaciones sociales son interdependientes y reaccionan entre si recíprocamente, adaptándose a los cam bios o procesos que se producen en otros segmentos de 
la sociedad, en forma tal que resulte asegurado el funcionamiento y la preservación del sistema (Rojas Soriano, 1983:58-59).

Las investigaciones derivadas de dicho enfoque, revelan aspectos parciales que bien sea favorecen la unidad y armonía social, o por el contrario, operan disfuncionalmente, mostrando cómo las condiciones de equilibrio se restablecen a través de cambios funcionales (Bernard, 1989:12).

En la mayoria de los casos, los partidarios de estos métodos que se pueden reunir bajo el nombre de descriptivos, incluso antes de comenzar el trabajo, implicitamente han tomado ya partido por el orden social actual, que consideran como natural y normal, y que ni siquiera les parece necesitar una justificación (Goldmann, Op. cit.:33).

El problema de la investigación social en el contexto del establecimiento científico tanto universitario como estatal, radica esencialmente en el fisicismo, 0 extensión al ámbito social, de la concepción fáctico-descriptiva de las ciencias naturales, la cual parte de la ecuación realidad $=$ fenómenos, e implicitamente asume que el estudio de lo social solo es ciencia en la medida en que hace abstracción de la dimensión axiológica de los hechos humanos (Delgado, 1987:10), omitiendo que ambos aspectos están presentes tanto en la naturaleza como en la sociedad.

Según 10 muestra W.T. Jones (1976) en su libro : Las Ciencias y las Humanidades, Conflicto y Reconciliación, la ciencia al igual que el arte o la literatura
(Humanidades), es un metalenguaje que expresa la unidad ontológica de una realidad constituida por hechos y valores, el cual debe ser evaluado en su eficacia, conforme a las reglas de preferencias de cada una de ellas : las ciencias, de acuerdo con su capacidad para describir los hechos, y las humanidades, a partir de su aptitud para expresar los valores.

En la perspectiva indicada, se hace patente que al pretender operar las ciencias sociales con las categorias de las ciencias naturales, es decir dentro de una óptica fisicista que implica orientar el foco de atención a los hechos en detrimento de los valores, castran el fenómeno humano reduciéndolo a su dimensión factual, omitiendo elementos de comprensión del mismo fundamentales para una adecuada aprehensión de las condiciones que lo determinan y haciendo imposible su inserción en una teoría capaz de esclarecer sus relaciones con el contexto social, económico y político (Goldmann, 1977:22-31). Estamos entonces en presencia de un factor de distorsión ideológica derivado de las relaciones de poder en las que se inscribe la ciencia dentro del sistema capitalista.

En efecto, la ciencia, y en particular, las ciencias físicas, como categoría histórica y superestructural, responden en su evolución al desarrollo de las fuerzas productivas y a las necesidades e intereses de las clases sociales dominantes dentro de la relaciones de producción que se generan a consecuen cia del mencionado desarrollo (Delgado, 1987:114); pero el cientifico que actúa enmarcado en dicho contexto material, no lo advierte al asumir de modo idealista, que como interés, el conocimiento es parte de "un movi- 
miento consciente del hombre hacia la realización de metas libremente escogidas, cuando en verdad el interés es objetivo por su origen, es decir, por el carácter objetivo de la necesidad que lo genera" (Delgado, 1987:111).

Como consecuencia, el científico social adherido a la visión positivista irradiada desde las ciencias físicas, carece de la perspectiva dialéctica necesaria para advertir el carácter histórico de las relaciones que describe, actuando como factor legitimador de un proceso cuyas relaciones internas describe a manera de leyes absolutas.

\section{Ciencia, cientificismo y compromiso social y político}

El problema del subdesarrollo es esencialmente de naturaleza política y responde a estructuras de poder externas e internas mediante las cuales la economla y la sociedad son movidas por fuerzas ajenas a su propia dinámica, estableciendo lazos de dependencia, en virtud de los cuales "la economía de ciertos países es condicionada por el desarrollo y la expan sión de otra economía a la cual la primera está supeditada..." (ChilcoteEdelstein, 1974:27).

En ese contexto, constituye una falacia y una forma de ocultamiento de las verdaderas causas del subdesarrollo, el señalar el atraso cientffico y tec nológico como generador del mismo, cuando constituye en reali dad una de sus consecuencias, derivándose de dicha falacia una de las deformaciones mas nefastas de dicha ideología, como lo es el cientificismo que "exalta las excelencias de la libertad de investigación, de la objetividad y neutralidad ideológica de la ciencia, y del desarrollo de la tecnología como único modelo válido de progreso y bienestar social" (Delgado, 1987:112).

Como consecuencia de ese manejo cientificista del problema del subdesarrollo, queda postergada la posibilidad efectiva de actuar en función de una estrategia dirigida a crear las condiciones para el desarrollo, como lo serían el establecimiento de políticas económicas y sociales que propendan a la conquista de una auténtica independencia nacional y a la redistribución de la riqueza.

Mientras tanto se frustan los esfuerzos supuestamente dirigidos a "ocuparse de problemas nacionales y a hacer ciencia aplicada of funcional" y por ejercer la cacareada libertad de investigación en función de los "intereses nacionales", por cuanto dentro de estructuras neo coloniales esos esfuerzos sirven para consolidar los intereses multinacionales asi como los de la burguesía nacional a los cuales aquellos están asociados, mediante la utilización de la capacidad científica y tecnológica interna en función de desarrollos destinados a mejorar el giro empresarial de dichas asociaciones (Delgado, 1987: 112-114).

Ahora bien, al referimos a la ciencia y la tecnologia como factores de desarroIlo, es necesario distinguir entre tecnologia física y tecnologia social (Varsavsky, 1972:29 y s). Esta última consiste tanto en los conocimientos teóricos necesarios como en la capacidad de operar a través de los mismos sobre la propia realidad social, económica y política, para la creación de las condiciones susceptibles de generar un desarrollo nacional independiente. 
La tecnología social debe estar basada en conocimientos suministrados interdisciplinariamente por las ciencias sociales, a objeto de esclarecerse el origen y la naturaleza de la dependencia en tanto raiz del subdesarrollo, así como de definir los aspectos estratégicos susceptibles de modificar los lazos neocoloniales, incidiendo a la vez en las causas de los mismos y en sus efectos, de manera a generar la transformación social, política y económica necesaria.

Por lo antes dicho, la Universidad de los paises del Tercer Mundo enfrenta una responsabilidad mas elevada que la de las naciones industrializadas en la medida en que constituye el único centro de producción de ciencia y cultura, y es en la correcta elección de las políticas cientificas de esas casas de estudios que radica la articulación ente Etica e Investigación, de tal forma que no sean creadas expectativas inviables respecto de las relaciones entre ciencia y desarrollo, ni que le sea reclamado a nuestras instituciones el cumplimiento de las funciones que les corresponden bien sea a la industria, bien sea al Estado.

Uno de los principales escollos para un claro entendimiento de esa situación, es de naturaleza ideológica, como lo constituye el argumento del establecimiento económico y político de que la Universidad debe dirigir sus prioridades a la investigación aplicada y al desarrollo tecnológico, sirviendo a la vez ese argumento de disuasivo a la investigación social cuyo enfoque dependentista 0 en todo caso crítico, perturba el funcionamiento neocolonial de nuestras sociedades, y de excusa a la incapacidad del sis- tema por alcanzar el anhelado equilibrio socio-económico.

Ante la imposibilidad de que la Universidad responda al desatío asi planteado, surge el chantaje de la ineficacia del trabajo universitario y el fantasma del recorte presupuestario que por otra vía, inhibe la investigación tanto básica como crítica, con el alegato de que ninguna de las dos contribuye al desarrollo nacional a la luz del criterio de pertinencia social manejado por los sectores de poder.

Se trata de un asedio sicológico destinado a crear "mala conciencia" en los universitarios e inducir en ellos una actitud de "servidumbre" hacia el mercado científico, con la consiguiente "renuncia a la preocupación por el significado social de su actividad, desvinculándola de los problemas políticos, y a la entrega de lleno a su 'carrera', aceptando para ello las normas y los valores de los grandes centros internacionales concretados en un escalafón... El seguidismo y el colonialismo son las características de esta incorporación de los científicos criollos a la élite empresarial de la ciencia..." (Delgado, 1987:113).

\section{Conclusiones}

El compromiso social y político del investigador en las ciencias sociales en una sociedad dependiente, reside en su capacidad por desarrollar una investigación que devele en todos los ámbitos, la ideología y actuación del neocolonialismo como forma de perpetuación de la iñicua división Norte- Sur (Bernard, 1995a). Se trata de una tarea que requiere independencia y lucidez, dos cualidades 
combatidas por el estable cimiento en la investigación universitaria, bien sea a través de su incorporación al giro económico de la sociedad, bien sea por el enfoque ideológico de la problemática científica (Delgado, 1987:113).

Como muestra de este último, la revista arbitrada surgida de una comunidad cientifica que se pretende "universal", aunque al decir de Varsavsky, esa "universalidad" "responde a un tipo de sociedad que domina casi todo el mundo: la sociedad de consumo, individualista-competitiva y burocratizada" (Delgado, 1989:19), como medio de difusión del avance del conocimiento, en particular en las ciencias naturales, la cual debe garantizar por via del "arbitraje", la seriedad y ética de los trabajos publicados, constituyéndose en un invalorable y generoso medio de comunicación para todos los investigadores a nivel mundial; representa para las ciencias sociales, un peligroso instrumento de distorsión de sus funciones.

En efecto, si admitimos, como lo hemos mostrado en este trabajo, que el investigador social está de alguna manera influenciado por los tres "ismos" señalados, como árbitro se convierte en expedito y eficiente filtro de cualquier planteamiento crítico, al garantizar la supuesta "pertinencia" social y política de las investigaciones publicadas.

Por consiguiente, el sentido de nuestro trabajo es el de llamar la atención sobre el riesgo de que a través del arbitraje y otras modalidades burocrático-cientificas, como la promoción de la carrera del investigador, los incentivos eco nómicos y reconocimientos por via de clasifica- ción, el financiamiento de proyectos, etc., sea oficializada y reforzada la concepción positivista, fisicista y cientificista como modelo para las ciencias sociales, convirtiéndose dichas iniciativas en formas de control ideológico por parte de los sectores de poder, los cuales, por ejemplo, han otorgado a la revista arbitrada el monopolio de la comunicación científica en el ámbito de las ciencias sociales.

En el sentido indicado, debe promoverse la resistencia de los cientifícos sociales, al yugo de la mencionada ideología, la cual promueve una visión estática y neutral del fenómeno social, justificando la postergación sine die de la transformación en las sociedades dependientes; debe asimismo exaltarse el compromiso social y político del investigador cuyo norte permanente es el de la construcción de la teoria dialéctica que permite avizorar ese cambio, y la creación de la tecnología social capaz de generarlo. En esa misión, la revista arbitrada debe liberarse de la "censura" y garantizar que el arbitraje no se convierta en "arbitrariedad" ideológica.

\section{Referencias bibliográficas}

Bernard, Brigitte (1978); Argumentos metodológicos para una investigación de las relaciones entre las ideologías revolución arias de 1789 y 1810 , Cuaderno de trabajo no. 25, IFD-LUZ, Maracaibo.

Bernard, Brigitte (1989); Estructura e Historia en las Principales Corrientes Metodológicas Contemporáneas en Ciencias Sociales: Funcionalismo, Estructuralismo y Marxismo, 2da. ed., Maracaibo, EDILUZ. 
Bernard, Brigitte (1995a); "Las relaciones en" tre ética e investigación en un mundo desigual", Sección Contrapalabra, La Universidad del Zulia, Maracaibo, LUZ, febrero de 1995.

Bernard, Brigitte (1995b); "Fundamentos epistemológicos del dualismo metodológico en el binomio ciencias naturales/ciencias sociales", Revista de FIlosofia, Vol. 22, no. 2, Centro de Estudios Filosóficos, Facultad de Hddes, y Educación, LUZ, Maracaibo.

Bunge, Mario (1980); Epistemología, Editorial Ariel, Barcelona.

Chilcote, Ronald H. y Edelstein, Joel C. (1974); Latin America : The Struggle with Dependency and Beyond, Cambridge, Mass. (USA), Schenkman Publishing Company Inc..

Cossio, Carlos (1959); EI Derecho en el Derecho Judicial, Ed. Abeledo-Perrot, Buenos Aires.

Delgado Ocando, J.M. (1981); "Ciencia, tecnología y progreso social", Ponencia presentada en "23 años de democracia en Venezuela - Jomadas de Análisis", Mérida, ULA, julio de 1981.

Delgado Ocando, J.M. (1987); Hipótesis para una Filosofía Antihegemónica del Derecho y del Estado, 2da. ed., IFDLUZ, Mara caibo.

Delgado Ocando, J.M. (1989); "La legitimidad como eficacia transformadora y su nivel psico-social de mediación", en GABAL DON, Luis Gerardo, Legitimidad y Sociedad, ULA - Altadil Ediciones, Caracas.

Delgado Ocando, J.M.: "Algunas reflexiones sobre clencia y politica", Maracaibo, IFD-LUZ, 1990.
De Sousa Santos, Boaventura (1996); "Ciencia, sociedad y Derecho frente al debate modemidad/postmodemidad", Revista Frónesis, Número Especial, IFD-LUZ, Maracaibo, Junio 1996.

Freund, Julien (1975); Las Teorias de las Clencias Humanas, Ed. Península, Barcelona (España).

Goldmann, Lucien (1977); trad. Por Josefina Martínez Alinari; Las Ciencias Humanas y la Filosofía, Ed. Nueva Visión, Buenos Aires.

Jones, W.T. (1976); Las Ciencias y las Humanidades; Conflicto y Reconciliación, México, F.C.E..

Méndez, Evaristo (1984); Qué y Cómo Investigar (mimeo), LUZ, Maracaibo.

Nuñez Tenorio (1972); Introducción a la Ciencia, Ed. C.M. Nueva Izquierda, Caracas.

Popper, Karl R. (1994); trad. por Néstor MIguez; Conjeturas y Refutación - EI Desarrollo del Conocimiento Clentífico, Ed. Paidós, Buenos Alres.

Rojas Soriano, Raul (1983); El Proceso de le Investigeción Científica, 3ra. ed., Ed. Trillas, México.

Sánchez Aranguren - Guarisma A., Jose Gerardo (s/f); Métodos de Investigación, Ediciones Eneva, Universidad Bicentenaria de Aragua.

Terrades, A. (1977); "La Revolución científco-técnica y los paises dependientes" en La Revolucion Cientifico-Técnica, Caracas, El Cid Editor.

Varsavsky, Oscar (1972); Hacia una Política Científica Nacional, Ed. Periferia, Buenos Aires. 